\title{
INFLUENCE OF SUBSTRATE TEMPERATURE ON MAGNETIC AND STRUCTURAL PROPERTIES OF RF MAGNETRON SPUTTERED Co-Cr THIN FILMS
}

Jong-Sung Baek, YaRk-Yeon Kim, Woo-Young Lim

Dept. of Phys., Korea Univ., Chochiwon 339-700, South Korea

YURNG-DAE KIM

Dept. of Sci. Edu., Chungbuk Nat'l Univ., Cheongju 360-763, South Korea

AND SEONG-Cho Yu

Dept. of Phys., Chungbuk Nat'l Univ., Cheongju 360-763, South Korea

The present paper describes the substrate temperature $T_{s}$ dependence on magnetic and structural properties of $\mathrm{Co}-21$ at.\% $\mathrm{Cr}$ thin films deposited on the Corning glass substrate by RF magnetron sputtering. The properties of the samples, such as saturation magnetization $M_{s}$, in-plane squareness ratio $S_{\|}$, effective magnetic anisotropy field $H_{k}^{\prime}$, spectroscopic splitting factor $g$, and hcp (002) peak intensity $I_{002}$, were examined by vibrating sample magnetometer, $Q$-band $(\approx 33.9 \mathrm{GHz}$ ) ferromagnetic resonance measurements and X-ray diffractometry. Specially, the presence of the various ferromagnetic constituents in $\mathrm{Co}-\mathrm{Cr}$ thin films are examined by the ferromagnetic resonance studies. With increasing $\mathrm{T}_{s}$ from room temperature to $200^{\circ} \mathrm{C}, S_{\|}$ increases from 0.08 to 0.56 but $H_{k}^{\prime}$ decreases from 2530 Oe to $-3900 \mathrm{Oe}$. Also $I_{002}$ decreases with increasing $T_{s}$. It is summarized that the perpendicular magnetic anisotropy and $c$-axis orientation of $\mathrm{Co}-\mathrm{Cr}$ films are improved at lower substrate temperature.

PACS numbers: $75.50 . \mathrm{Ss}$

\section{Introduction}

Co-Cr thin films, one of the most promising media for the perpendicular magnetic recording system, have been extensively studied [1]. It is known that large perpendicular magnetic anisotropy in $\mathrm{Co}-\mathrm{Cr}$ thin film is essential for the perpendicular magnetic recording media with ultrahigh density. The magnetic and structural properties of sputtered $\mathrm{Co}-\mathrm{Cr}$ thin films, such as full width at half maximum $\Delta \theta_{50}$, in-plane squareness ratio $S_{\|}\left(=M_{\mathrm{r}} / M_{\mathrm{s}}\right)$, perpendicular coercivity $H_{\mathrm{c} \perp}$, and magnetic anisotropy field $H_{k}$, are strongly dependent on the sputtering 
parameters, such as substrate temperature, argon pressure, and sputtering power, etc. $[2,3]$.

In this paper $\mathrm{Co}-\mathrm{Cr}$ thin films were prepared at various substrate temperature. The dependence of the magnetic and structural properties on $T_{\mathbf{s}}$ were examined by a vibrating sample magnetometer (VSM), $Q$-band $(\approx 33.9 \mathrm{GHz})$ ferromagnetic resonance (FMR) measurements and X-ray diffractometry.

\section{Experimental}

Co-21 at.\%Cr thin films were deposited on Corning glass substrate by RF magnetron sputtering system (ANELVA, model SPF-210) at room temperature, $50^{\circ} \mathrm{C}, 100^{\circ} \mathrm{C}, 150^{\circ} \mathrm{C}$, and $200^{\circ} \mathrm{C}$, respectively. Alloy target of 4 inch diameter was used. The background pressure was lower than $5.0 \times 10^{-7} \mathrm{Tr}$ and argon with purity $99.999 \%$ was used as a sputtering gas. The deposition rate was kept at $140 \AA / \mathrm{min}$ with the RF high voltage of $1.6 \mathrm{kV}$ and the argon pressure of $25 \mathrm{mTr}$. The film thickness was about $5600 \AA$. The substrate holder was water cooled while $T_{\mathrm{s}}$ was kept at room temperature. For the experiments varying $T_{\mathbf{s}}$, it was heated by a heater mounted behind the substrate holder base. $T_{\mathbf{s}}$ was measured by a thermocouple attached to the substrate holder surface. $S_{\|}$were determined from the in-plane hysteresis loops which were measured by a VSM at room temperature. Crystallographic characteristics of $\mathrm{Co}-\mathrm{Cr}$ films were analyzed by X-ray diffractometry. In order to investigate the effective magnetic anisotropy fields $H_{k}^{\prime}$ and the presence of the various ferromagnetic constituents in Co-Cr thin films, $Q$-band $(\approx 33.9 \mathrm{GHz})$ ferromagnetic resonance measurements were performed. FMR measurements were carried out using a commercial Brucker EPR spectrometer (ESP 300 Series) at room temperature. The FMR spectra were obtained for various orientations of the applied DC magnetic field, from perpendicular to in-plane direction (angle step $10^{\circ}$ ).

\section{Results and discussion}

Figure 1 represents $S_{\|}$as a function of $T_{\mathbf{s}}$ for the films. The film deposited at room temperature shows $S_{\|}$of 0.08 . With increasing substrate temperature, $S_{\|}$ increases to 0.56 . Figure 2 represents $I_{002}$ as a function of $T_{s} . I_{002}$ has a maximum value at room temperature, and decreases with increasing substrate temperature. Figure 3 shows the FMR spectra of the samples sputtered at room temperature, $50^{\circ} \mathrm{C}, 100^{\circ} \mathrm{C}, 150^{\circ} \mathrm{C}$, and $200^{\circ} \mathrm{C}$, respectively. The FMR spectra were analysed after the following resonance condition [4]:

$$
\left(\frac{\omega}{\gamma}\right)^{2}=\left(H+H_{k}^{\prime} \sin ^{2} \phi_{H}\right)\left(H-H_{k}^{\prime} \cos 2 \phi_{H}\right),
$$

where $\gamma$ is the gyromagnetic ratio, $H_{k}^{\prime}\left(=H_{k}-4 \pi M_{s}\right)$ is the effective magnetic anisotropy field, and $\phi_{H}$ is the angle between the applied DC magnetic field and the film plane.

In Fig. 3a, the signal which appeared in the spectrum of parallel resonance $\left(\phi_{H}=0^{\circ}\right)$, moves toward lower DC magnetic field with increasing $\phi_{H}$. Accordingly, it is considered that the signal represents a positive anisotropy resonance $\left(H_{k}^{\prime}=2530 \mathrm{Oe}\right)$ [5]. The letter $P$ in the figure stands for this. The weak signal, 


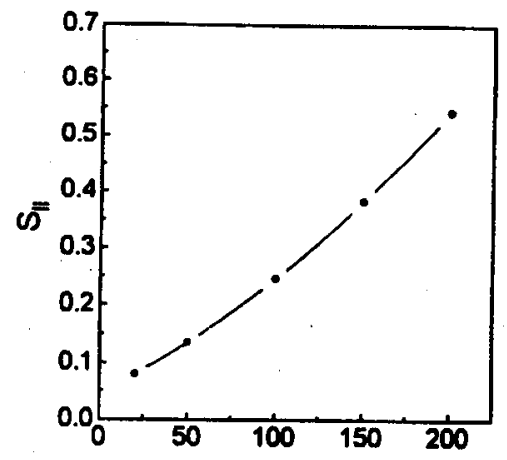

Fig.1

$T_{s}(\mathrm{c})$

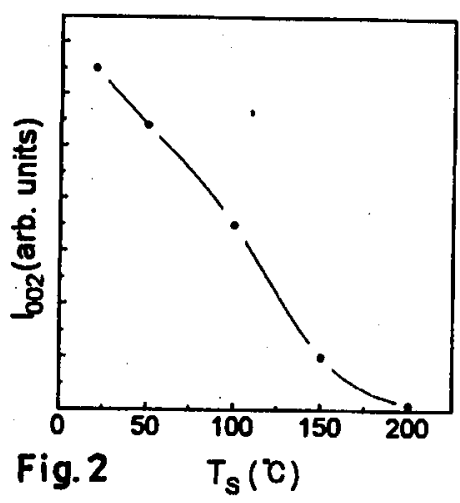

Fig. 1. $\mathrm{S}_{\|}$as a function of $T_{\mathrm{s}}$.

Fig. 2. $I_{002}$ as a function of $T_{s}$.

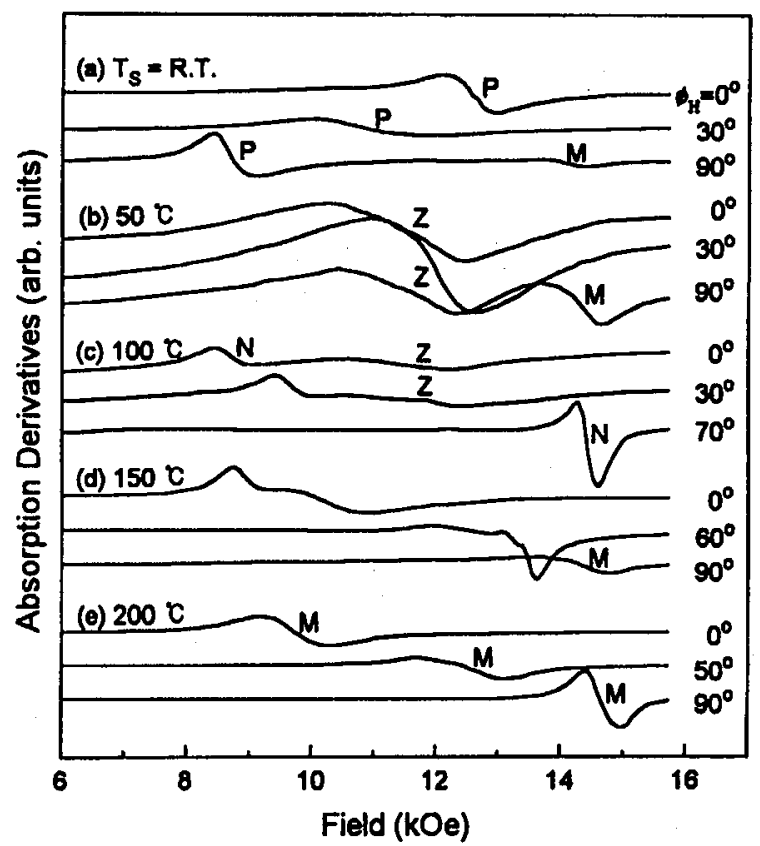

Fig. 3. The FMR spectra of Co-Cr thin films ( $P$ - positive anisotropy resonance, $M-$ $4 \pi M_{\mathrm{s}}$ resonance, $Z$ - zero anisotropy resonance, $N$ - negative anisotropy resonance).

which appeared in the spectrum of perpendicular resonance $\left(\phi_{H}=90^{\circ}\right)$ at higher DC magnetic field, vanishes with decreasing $\phi_{H}$. Considering Eq. (1), it seems $4 \pi M_{\mathrm{s}}$ resonance $\left(H_{k}^{\prime}=-4 \pi M_{\mathrm{s}}\right)$. Because the positive anisotropy resonance signal is dominant in this diagram, it is considered that the sample fabricated at room temperature has large perpendicular magnetic anisotropy. In the case of $T_{\mathrm{s}}$ of $50^{\circ} \mathrm{C}$, the angular independent signal (zero anisotropy resonance: $H_{k}^{\prime} \approx 0$ ) and 
$4 \pi M_{\mathrm{s}}$ resonance appeared together, as shown in Fig. $3 \mathrm{~b}$. In the substrate temperature of $100^{\circ} \mathrm{C}$, negative anisotropy resonance $\left(H_{k}^{\prime}<0\right)$ and $4 \pi M_{\mathrm{s}}$ resonance appeared together but negative anisotropy resonance signal dominates, as shown in Fig. 3c. In Fig. 3d, the mixed signal appeared in the spectrum of parallel resonance $\left(\phi_{H}=0^{\circ}\right)$. Only $4 \pi M_{\mathrm{s}}$ resonance $\left(H_{k}^{\prime}=-4 \pi M_{\mathrm{s}}=-3900 \mathrm{Oe}\right)$ was observed in the FMR spectra of the film deposited $200^{\circ} \mathrm{C}$, as shown in Fig. 3e. $g$ values are about $2.22( \pm 0.07)$ within the ranges of varied substrate temperature. It is observed that the positive anisotropy signal diminishes as the substrate temperature increases, but the $4 \pi M_{\mathrm{s}}$ resonance signal and the negative anisotropy resonance signal grow as the substrate temperature increases. Hence it is considered that the perpendicular magnetic anisotropy and $c$-axis orientation of $\mathrm{Co}-\mathrm{Cr}$ films are improved at lower substrate temperature, but deteriorated at higher $T_{\mathrm{s}}$. These results are in agreement with previous studies $[2,3]$.

\section{Conclusion}

In this paper the influence of the substrate temperature on the magnetic and structural properties of $\mathrm{Co}-21$ at.\% $\mathrm{Cr}$ thin films deposited on Corning glass substrate was presented. Analyzing VSM experiments and FMR spectra, we observed that the positive anisotropy diminishes as the substrate temperature increases. We also observed magnetically non-identical regions in morphology. It is summarized that the perpendicular magnetic anisotropy and $c$-axis orientation of $\mathrm{Co}-\mathrm{Cr}$ films are improved at lower substrate temperature.

\section{Acknowledgment}

This work was supported by the Basic Science Research Institute Program, Korea Ministry of Education 1995, Project No. 2410 and KOSEF Program, Project No. 94-0300-07-01-3.

\section{References}

[1] S. Kadokura, M. Naoe, IEEE Trans. Magn. MAG-18, 1113 (1982).

[2] J.C. Lodder, T. Wielinga, IEEE Trans. Magn. MAG-20, 57 (1984).

[3] Y. Uchiyama, H. Sato, Y. Kimamoto, IEEE Trans. Magn. MAG-28, 2010 (1992).

[4] C. Kittel, Phys. Rev. 73, 155 (1948).

[5] P.V. Michell, A. Layadi, N.S. VanderVen, J.O. Artman, J. Appl. Phys. 57, 3976 (1985). 